\title{
An Efficient 2-D MLFMM-UTD Hybrid Method to Model Non-Line-of-Sight Propagation
}

\author{
Giorgos Karagounis, Daniël De Zutter*, and Dries Vande Ginste*
}

\begin{abstract}
We present a hybrid method that combines the Multilevel Fast Multipole Method (MLFMM) with the Uniform Theory of Diffraction (UTD) to model two-dimensional (2-D) scattering problems. The method is especially suited to model scattering in the presence of very large scatterers that obstruct the line-of-sight propagation between different devices with a more intricate geometry, such as antennas. The discretization of the large scatterers is avoided by using ray-based methods. An $O(n)$ scaling is achieved for the computational time and required memory, $n$ being the number of unknowns needed to discretize the antennas. The method is validated by a numerical example.
\end{abstract}

\section{INTRODUCTION}

Scattering problems in piecewise homogeneous domains are modeled by solving the boundary integral equations (BIEs) on the boundary surface between domains. The tangential currents on these boundaries are discretized, which results in a Method of Moments (MoM) scheme with $N$ unknowns and $O\left(N^{2}\right)$ computational complexity. Nowadays, Multilevel Fast Multipole Methods (MLFMM) are employed to reduce the computational complexity to $O(N \log N)$. Moreover, these methods are scalable over different processors, allowing to model complex geometries with billions of unknowns [1] on high-performance computing (HPC) infrastructures. A different way to tackle the problem is to efficiently account for the presence of large structures with canonical shapes by using ray-based methods such as geometrical optics (GO). Hybrid schemes combining the MoM and the Geometrical Theory of Diffraction (GTD) [2] or the MLFMM and the Uniform Theory of Diffraction (UTD) [3] considerably reduce the required memory and computational time.

We propose a novel hybrid MLFMM-UTD method that accurately describes the fields in the case of non line-of-sight (NLoS) communication. Besides significantly lowering the number of unknowns by avoiding the discretization of the scatterers that can be treated by UTD, the method has computational complexity $O(n)$ for relevant structures such as an-

\footnotetext{
${ }^{*}$ IBCN/Electromagnetics Group, Department of Information Technology, Ghent University/iMinds, SintPietersnieuwstraat 41, 9000 B-Gent, Belgium. E-mail: giorgos.karagounis@UGent.be
}

tennas, $n$ being the number of unknowns needed to discretize those antennas. Our goal is to illustrate the good accuracy of the method in the shadow of large bodies, while a limited amount of computational resources is required.

This contribution is organized as follows. In Section 2, the formalism is presented. In Section 3, the numerical complexity and the accuracy of the algorithm are investigated through illustrative examples. In Section 4, we present some concluding remarks.

Here, we deal with 2-D transverse magnetic (TM) problems. The z-axis is chosen as the axis of invariance. An $\exp (j \omega t)$ time dependence, with $\omega$ being the angular frequency, is assumed and suppressed throughout the text.

\section{FORMALISM}

After discretizing the antennas, constituting the BIE part of the problem, an MoM system with $n$ equations and $n$ unknows of the following form is derived:

$$
\boldsymbol{V}=\boldsymbol{Z}^{\text {tot }} \cdot \boldsymbol{I}=\boldsymbol{Z}^{\text {free }} \cdot \boldsymbol{I}+\boldsymbol{Z}^{r e f l} \cdot \boldsymbol{I}+\boldsymbol{Z}^{\text {diff }} \cdot \boldsymbol{I}
$$

in which $\boldsymbol{V}$ contains excitation terms, $\boldsymbol{I}$ is the unknown current vector and $\boldsymbol{Z}^{\text {tot }}$ is the so-called interaction matrix. In a classical MoM scheme, only $\boldsymbol{Z}^{\text {free }} \cdot \boldsymbol{I}$ is present in the right-hand side (RHS) and accounts for the free-space interactions between different BIE segments. To take the presence of the UTD scatterers into account, terms are added to the interaction matrix, such as reflection terms $\left(\boldsymbol{Z}^{\text {refl }} \cdot \boldsymbol{I}\right)$ and diffraction terms $\left(\boldsymbol{Z}^{\text {diff }} \cdot \boldsymbol{I}\right)$. In our method, each of the matrix-vector products (MVPs) needed to iteratively solve the problem is treated efficiently by the MLFMM.

The first MVP is performed by a traditional MLFMM scheme. A good introduction to MLFMM is found in [4]. Consider for example the geometry in Fig. 1. The discretized segments inside box $B^{\prime}$ interact with the segments inside box $B$. The MVP $\boldsymbol{Z}^{\text {free }} \cdot \boldsymbol{I}$ is decomposed in a three-step procedure according to MLFMM. At the lowest level, the currents $J_{s_{i}}$ of sources $s_{i}$ inside box $B^{\prime}$ are aggregated into outgoing plane waves $(O P W \mathrm{~s})$ at angles $\phi_{q^{\prime}}=2 \pi q^{\prime} /(2 Q+1)\left(q^{\prime}=-Q, \ldots, Q\right)$ as follows: 


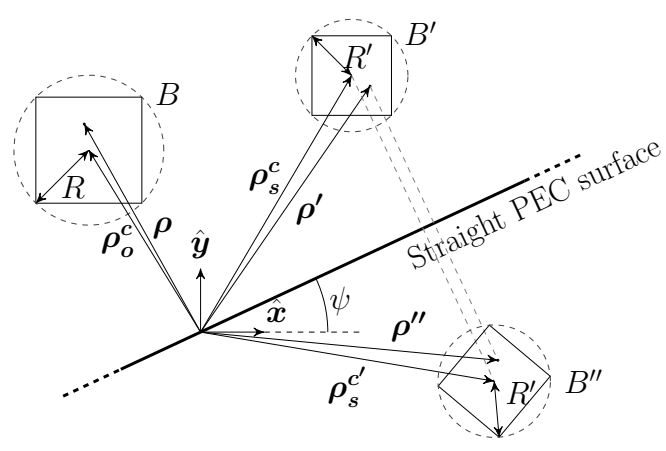

Figure 1: Illustration of the interaction between two MLFMM boxes. The PEC surface makes an angle $\psi$ with the $x$-axis. The image of a source $\boldsymbol{\rho}^{\prime}$ in source box $B^{\prime}$ is $\rho^{\prime \prime}$ in image box $B^{\prime \prime}$.

$$
O P W_{q^{\prime}}^{B^{\prime}}=\sum_{s_{i}} e^{j \boldsymbol{k}\left(\phi_{q^{\prime}}\right) \cdot\left(\boldsymbol{\rho}^{\prime}-\boldsymbol{\rho}_{s}^{c}\right)} J_{s_{i}},
$$

where $\boldsymbol{k}\left(\phi_{q^{\prime}}\right)=k\left(\cos \phi_{q^{\prime}} \hat{\boldsymbol{x}}+\sin \phi_{q^{\prime}} \hat{\boldsymbol{y}}\right)$ and $k$ is the wavenumber. $O P W \mathrm{~s}$ for higher-level boxes are found through interpolation and translation of the $O P W \mathrm{~s}$ of the lower level boxes they encompass, which is efficiently done by Fast Fourier Transforms (FFTs). Second, these $O P W \mathrm{~s}$ are translated into incoming plane waves $(I P W \mathrm{~s})$ at box $B$ by using:

$$
I P W_{q}^{B}=\sum_{q^{\prime}=-Q}^{Q} T_{q q^{\prime}} O P W_{q^{\prime}}^{B^{\prime}},
$$

where $T_{q q^{\prime}}$ are elements of the well-known (diagonal) translator of MLFMM and depend on the vector $\rho_{o}^{c}-\rho_{s}^{c}$ connecting the centers of the boxes. Then, these $I P W \mathrm{~s}$ are recursively anterpolated to yield the total $I P W \mathrm{~s}$ at the lowest level. Third, the $I P W \mathrm{~s}$ at the lowest level are disaggregated by the inverse procedure of (2) and the final result for the MVP is obtained.

The segments of the boxes in Fig. 1 also interact through reflection at the perfect electrically conducting (PEC) surface. In an MLFMM setting, the radiation pattern of image box $B^{\prime \prime}$ is to be translated to box $B$. The translation matrix is the traditional MLFMM translator but now depends on the vector $\boldsymbol{\rho}_{\boldsymbol{o}}^{\boldsymbol{c}}-\boldsymbol{\rho}_{\boldsymbol{s}}^{\boldsymbol{c}^{\prime}}$. Moreover, by using (2) and some basic vector algebra, it can be shown that

$$
O P W_{q^{\prime}}^{B^{\prime \prime}}=-\mathcal{R}_{2 \psi} O P W_{-q^{\prime}}^{B^{\prime}},
$$

where $\mathcal{R}_{2 \psi}$ denotes the rotation operation over an angle $2 \psi$. Such rotations are accurately and efficiently computed by FFTs [5].

The third MVP in the RHS of (1) corresponds to diffractions at edges, e.g. as shown in Fig. 2. To

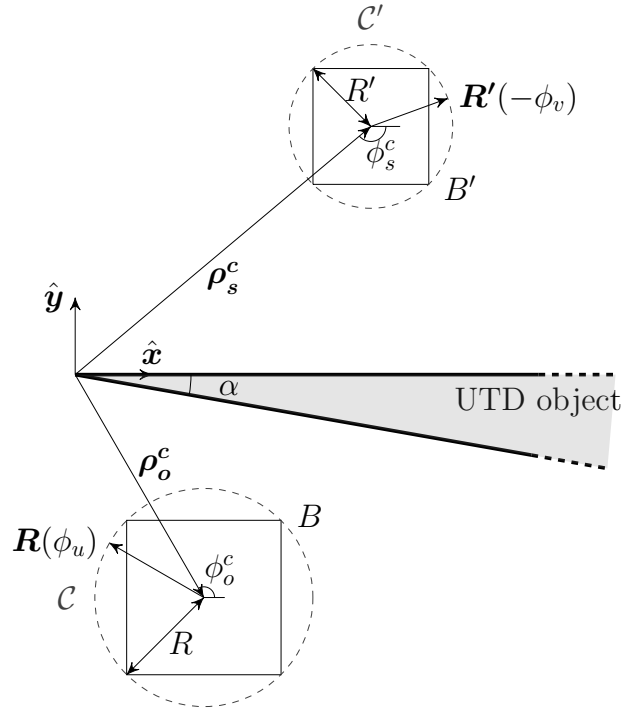

Figure 2: Illustration of the MLFMM-UTD geometry, where the UTD object is a PEC wedge with opening angle $\alpha$ situated between the MLFMM boxes.

this end, a new non-diagonal MLFMM translator has been derived, based on the method described in [6] that generalizes the use of UTD for arbitrary illumination sources and describes the field over extended regions of space. The source field leaving box $B^{\prime}$ and the diffracted field inside box $B$ are both expanded into multipoles. The harmonics of the two expansions are coupled through coefficients $t_{q q^{\prime}}$, given by

$$
\begin{gathered}
t_{q q^{\prime}}=\frac{1}{J_{q}(k R) J_{q^{\prime}}\left(k R^{\prime}\right)} \frac{1}{(4 Q+1)\left(4 Q^{\prime}+1\right)} \\
\sum_{u=-2 Q}^{2 Q} \sum_{v=-2 Q^{\prime}}^{2 Q^{\prime}} e^{-j q \phi_{u}} e^{-j q^{\prime} \phi_{v}} \frac{e^{-j k\left\|-\boldsymbol{\rho}_{o}^{c}+\boldsymbol{R}\left(\phi_{u}\right)\right\|}}{\sqrt{\left\|\boldsymbol{\rho}_{\boldsymbol{o}}^{\boldsymbol{c}}+\boldsymbol{R}\left(\phi_{u}\right)\right\|}} \\
\cdot D_{U T D}\left(L ; \boldsymbol{R}^{\prime}\left(-\phi_{v}\right), \boldsymbol{R}\left(\phi_{u}\right)\right) \\
\cdot H_{0}^{(2)}\left(k\left\|\boldsymbol{\rho}_{\boldsymbol{s}}^{\boldsymbol{c}}+\boldsymbol{R}^{\prime}\left(-\phi_{v}\right)\right\|\right),
\end{gathered}
$$

where $D_{U T D}$ and $L$ are the diffraction coefficient and the length parameter for line source illumination respectively (see [7]) and $Q^{\prime}$ and $Q$ depend on the radii $R^{\prime}$ and $R$ resp. (see Fig. 2). These coupling coefficients stem from a 2-D FFT of the sampled values of the diffracted field for line sources on the contour $\mathcal{C}^{\prime}$, observed along contour $\mathcal{C}$.

The multipole expansions can be converted into an equivalent spectral description, which is then identified to the radiation pattern of the MLFMM boxes. Finally, the elements $\tilde{T}_{p p^{\prime}}$ of the new MLFMM diffraction translator are given by 


$$
\begin{aligned}
& \tilde{T}_{p p^{\prime}}=\frac{1}{(2 Q+1)\left(2 Q^{\prime}+1\right)} . \\
& \sum_{q=-Q}^{Q} \sum_{q^{\prime}=-Q^{\prime}}^{Q^{\prime}} e^{j q\left(\phi_{p}+\pi / 2\right)} t_{q q^{\prime}} e^{-j q^{\prime}\left(\phi_{p^{\prime}}+\pi / 2\right)},
\end{aligned}
$$

where $p^{\prime}=-Q^{\prime}, \ldots, Q^{\prime}$ and $p=-Q, \ldots, Q$. These translation coefficients can also be efficiently computed by a 2-D FFT.

Similar to traditional MLFMMs, our algorithm is only used for pairs of boxes that are far from the diffraction tip. By leveraging an adaptive scheme, which allows higher-level boxes, that are further away from the diffraction tip, to interact with lower-level boxes, that reside closer to it, an $O(n)$ computational complexity for compact volume scatterers is achieved.

\section{EXAMPLES}

\subsection{Efficiency test}

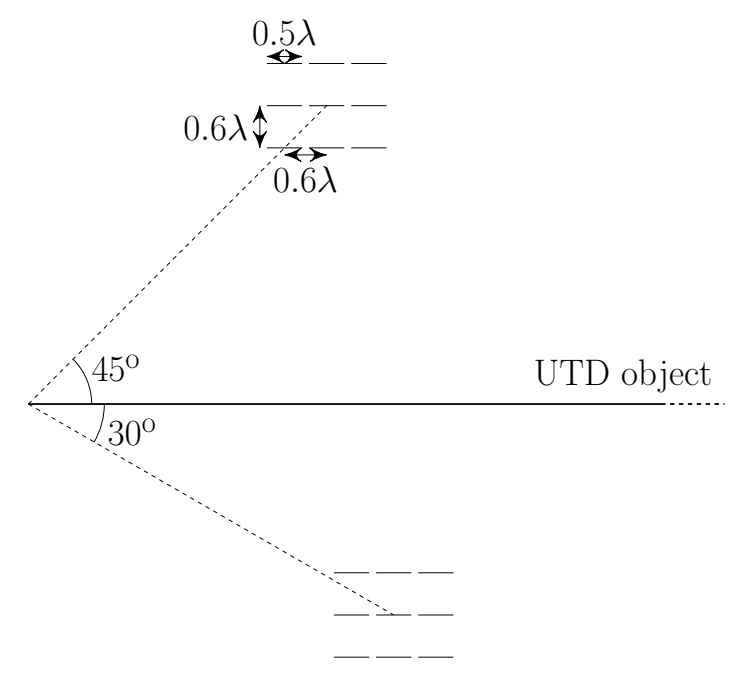

Figure 3: Geometry for the efficiency test with $m=3$.

The increase of computational resources with increasing number of unknowns is investigated for the configuration of two square $m \times m$ arrays of PEC strips separated by a long, straight PEC plate scatterer as shown in Fig. 3. The distance between the closest point of each array to the diffraction tip is kept fixed to $10 \lambda, \lambda$ being the wavelength. The number of unknowns is increased from 10 to 1332250 by increasing $m$.

The scaling of the CPU time during setup, the average MVP time and the memory requirements for our method and a traditional MoM-UTD scheme [2]

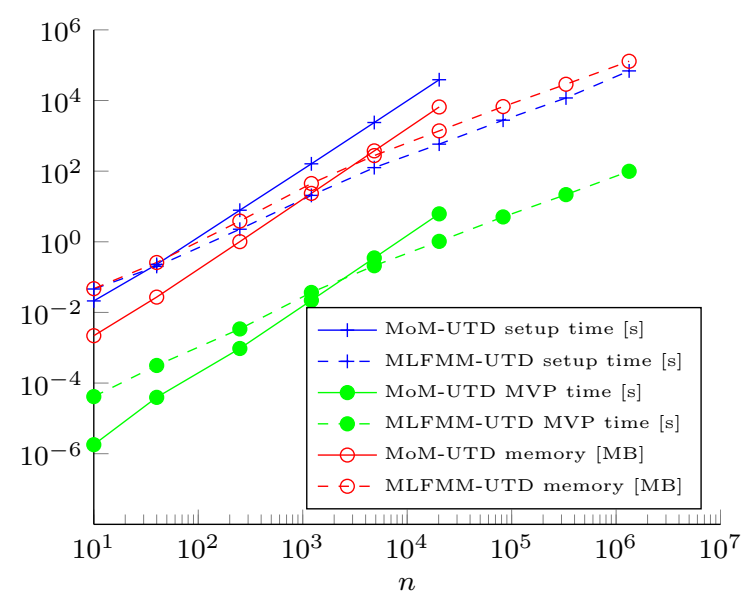

Figure 4: Scaling of the resources for an increasing number of unknowns $n$.

are summarized in Fig. 4. Our MLFMM-UTD hybrid scheme is much faster than the MoM-UTD during setup time even for a very low number of unknowns, due to the dominant computational load for the computation of the diffraction coefficients. For the MVP time and the required memory, the cross-over point is situated at 2500 unknowns, which is in line with conventional MLFMM solvers. Moreover, the $O(n)$ complexity is clearly obtained.

\subsection{Accuracy test}

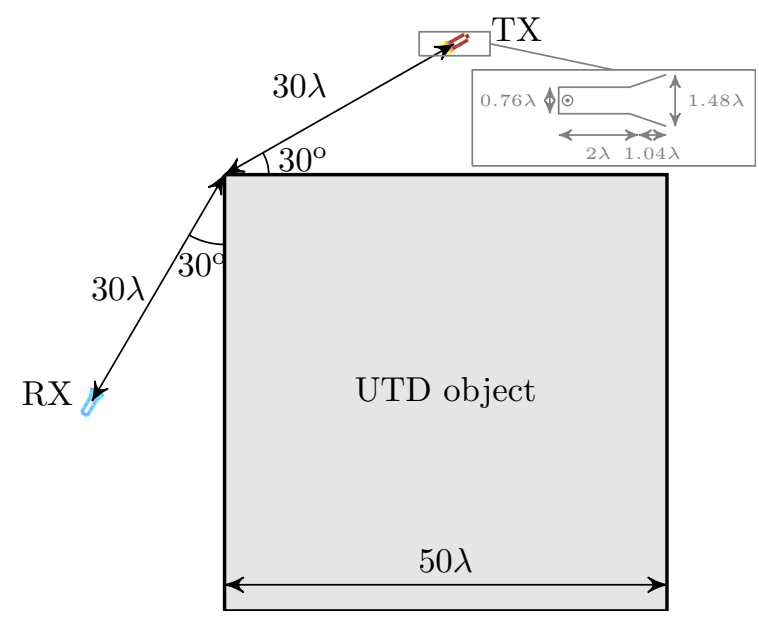

Figure 5: Geometry for the accuracy test. The induced currents on the horns are shown in color.

The accuracy of the method is validated for the example in Fig. 5. A transmitting horn antenna (TX) is placed at $30 \lambda$ from the upper left corner of a large square PEC scatterer, at an angle of $30^{\circ}$ w.r.t. the upper face. A receiving horn (RX) is placed in the 
shadow of the large scatterer, at $30 \lambda$ from the corner and at an angle of $30^{\circ}$ w.r.t. the left face. A $\lambda / 15$ discretization leads to $n=210$ unknowns. A line source with current strength $1 \mathrm{~A} / \mathrm{m}$ is placed along the symmetry axis of the TX, at $\lambda / 4$ of its back-end. The diffractions by all pertinent edges of the PEC square are superimposed in (5). Doubly diffracted rays are also taken into account by the diffraction coefficient presented in [8]. The currents are compared to these obtained by a traditional MLFMM solver by also discretizing the large square cilinder, leading to 2000 extra unknowns.

The MLFMM solver required 10 minutes to solve the system and 23.7 MB of memory. In contrast, the MLFMM-UTD method required only $0.7 \mathrm{~s}$ of solution time and 1.9 MB of memory. The amplitude of the currents is illustrated in Fig. 5. The relative error ( $\mathrm{RE})$, defined as

$$
R E_{i}=\left|\frac{J_{i}-J_{i, r e f}}{J_{i, r e f}}\right|,
$$

where $J_{i}$ and $J_{i, r e f}$ are the currents on the $i^{\text {th }}$ segment computed by MLFMM-UTD and MLFMM resp., is plotted in Fig. 6. A relative error of $1 \%$ is obtained for the currents on the RX, indicating that also the phase is modeled well by our method, this in contrast to other hybrid schemes, such as [3].

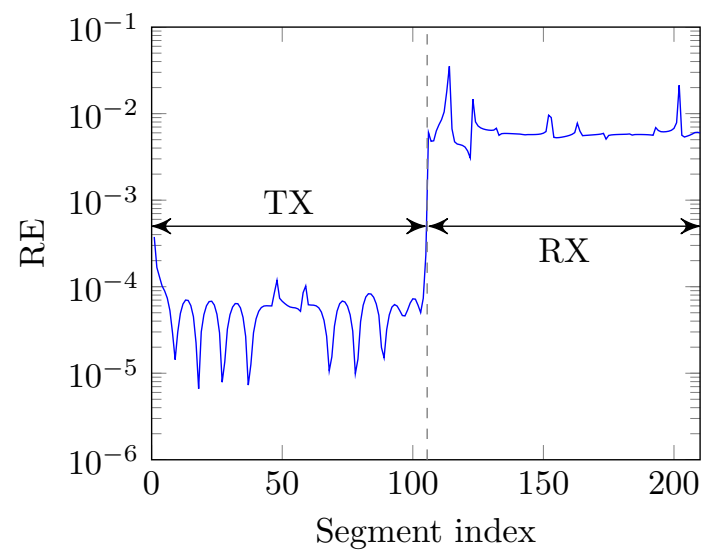

Figure 6: Relative error on the currents compared to an MLFMM solution.

\section{CONCLUSIONS}

We presented a hybrid 2-D MLFMM-UTD method that is suitable for non line-of-sight applications. The accuracy on the current compared to a fullwave simulation was about $1 \%$ in the shadow region. The computational complexity of the MLFMM-UTD method is $O(n)$ for an increasing number of unknowns $n$. The cross-over point compared to the classical MoM-UTD scheme was found at 2500 unknowns, which is in line with conventional MLFMM solvers.

\section{Acknowledgments}

We kindly thank the Research Foundation Flanders (FWO) for financially supporting this research.

\section{References}

[1] B. Michiels, J. Fostier, I. Bogaert, and D. De Zutter, "Full-Wave Simulations of Electromagnetic Scattering Problems With Billions of Unknowns," IEEE Trans. Antennas Propagat., vol. 63, no. 2, pp. 796-799, 2015.

[2] G. A. Thiele and T. H. Newhouse, "A Hybrid Technique for Combining Moment Methods with the Geometrical Theory of Diffraction," IEEE Trans. Antennas Propagat., vol. 23, no. 1, pp. 62-69, 1975.

[3] A. Tzoulis and T. F. Eibert, "A Hybrid FEBI-MLFMM-UTD Method for Numerical Solutions of Electromagnetic Problems Including Arbitrarily Shaped and Electrically Large Objects," IEEE Trans. Antennas Propagat., vol. 53, no. 10, pp. 3358-3366, 2005.

[4] W. C. Chew, J.-M. Jin, E. Michielssen, and J. Song, Fast and Efficient Algorithms in Computational Electromagnetics. Artech House Publishers, 2001.

[5] D. Vande Ginste, E. Michielssen, F. Olyslager, and D. De Zutter, "A High-Performance Upgrade of the Perfectly Matched Layer Multilevel Fast Multipole Algorithm for Large Planar Microwave Structures," IEEE Trans. Antennas Propagat., vol. 57, no. 6, pp. 1728-1739, 2009.

[6] G. Karagounis, D. De Zutter, and D. Vande Ginste, "Computation of the Diffraction from Complex Illumination Sources in Extended Regions of Space," $O p$ tics Express, vol. 21, no. 25, pp. 30 379-30391, 2013.

[7] R. G. Kouyoumjian and P. H. Pathak, "A Uniform Geometrical Theory of Diffraction for an Edge in a Perfectly Conducting Surface," Proceedings of the IEEE, vol. 62, 1448-1461, 1974.

[8] R. Tiberio, G. Manara, G. Pelosi and R. G. Kouyoumjian, "High-Frequency Electromagnetic Scattering of Plane Waves from Double Wedges," IEEE Trans. Antennas Propagat., vol. 37, no. 9, pp. 301172-1180, 1989. 